\title{
Präventive Arbeitsgestaltung in der digitalen Produktion: Ein Blick in die Kulissen
}

\author{
Anja Gerlmaier
}

\section{Digitalisierung als Herausforderung für Prävention und gesunde Arbeit}

Gegenwärtig planen immer mehr Industrieunternehmen smarte Formen von Automatisierung einzuführen, um individualisierte Produkte, erweiterte Geschäftsmodelle, kleine Losgrößen oder marktinduzierte starke Auftragsschwankungen anzugehen. Mit digitalen, zum Teil auf künstlicher Intelligenz basierenden, Fertigungsverfahren, Servicerobotern oder dem verstärkten Einsatz von Sensorik wird in der Regel das Ziel verfolgt, eine neue Stufe der Rationalisierung und hierdurch erhebliche Flexibilitäts- und Kostenvorteile im globalen Wettbewerb zu erzielen. Ein Blick hinter die Kulissen der Industrie 4.0-Kulisse zeigt, dass viele Industrieunternehmen bei der Einführung neuer digitaler Technologien derzeit oft keinen Bruch der gewachsenen technisch-organisatorischen und personellen Strukturen vollziehen (Hirsch-Kreinsen 2018). Anstelle cyberphysischer Systeme werden in den meisten Produktionsbereichen Insellösungen vorgefunden, in denen etwa mit fahrerlosen Transportsystemen, RFID-Technik oder KI-gestützten Planungs- und Steuerungssystemen experimentiert wird (Georg, Katenkamp und Guhlemann 2017). Die Technikeinführung gestaltet sich hierbei nicht selten als „Operation am offenen Herzen“, weil bewährte Produktionsprozesse und -routinen mit ungewissem Ausgang teilweise in der laufenden Produktion durchbrochen werden. Dies stellt nicht nur eine technische, sondern auch eine erhebliche Herausforderung für die Sicherheit und den Gesundheitsschutz der davon betroffenen Beschäftigten dar: neben neuartigen Unfallgefahren können durch veränderte Rollen- und Aufgabenzuschnitte bzw. Qualifikationsanforderungen Fehlbeanspruchungen wie Stress oder Monotonie resultieren, wenn Kriterien menschengerechter Arbeitsgestaltung nicht berücksichtigt werden.

Digitale Transformationen bergen aber auch erhebliche Chancen für eine humanzentrierte Gestaltung von Arbeit: der Einsatz adaptiver Assistenzsysteme bietet etwa die Möglichkeit der Berücksichtigung unterschiedlicher Qualifikationsniveaus und physiologischer Leistungsvoraussetzungen unterschiedlicher Beschäftigtengruppen. Mobile ITK-Geräte können 
Arbeitsprozesse erleichtern und flexiblere Formen von Arbeiten und Leben ermöglichen (Zimmermann 2017). Insbesondere dem Einsatz von Sensorik und auf künstlicher Intelligenz basierender Systeme werden darüber hinaus erhebliche Chancen für eine Reduzierung monotoner Arbeitsaufgaben und physikalischer Beanspruchungen zugeschrieben, die sich wiederum günstig auf den Verbleib älterer Beschäftigter im Erwerbsleben auswirken sollte (DGUV 2017). Bisher gibt es aber kaum arbeitswissenschaftlich fundierte Feldstudien, in denen positive gesundheitliche Effekte nach der Einführung neuer Digitaltechnik festgestellt werden konnten. Studien über Belastungs- und Gesundheitswirkungen, beispielsweise zur Einführung ganzheitlicher Produktionssysteme, weisen eher auf erhöhte Beanspruchungsrisiken hin (vgl. Gerst 2011). Stellt die fortschreitende Digitalisierung einen erfolgversprechenden Treiber für sichere, gesunde und lernförderliche Arbeit dar? Oder birgt sie das Risiko einer weiteren Verschärfung einer ohnehin von vielen Beschäftigten als zu hoch erlebten Arbeitsintensität? Im Rahmen arbeitspolitischer Diskurse finden sich zu dieser Fragestellung durchaus unterschiedliche Einschätzungen: in Positionspapieren der Arbeitgeberseite wird davon ausgegangen, dass die zunehmende Vernetzung von Produktionssystemen nur mit einem hohen Maß an dezentraler Selbstorganisation und agilen Führungs- und Kooperationsformen beherrschbar sei und sich deswegen innovative und präventiv ausgerichtete Arbeits- und Organisationsformen quasi naturwüchsig durchsetzen würden (BDA 2015). Einige Industriesoziolog*innen gehen wiederum davon aus, dass Industrie 4.0 für bestimmte Beschäftigtengruppen mit schwer ersetzbarem Know-how einen Push-Faktor darstellt, um erweiterte Ansprüche an die Arbeitsqualität geltend machen zu können (u.a. Kratzer 2018; Heidenreich, Kirch und Mattes 2008). Diesen Einschätzungen stehen allerdings auch Befürchtungen - insbesondere der Gewerkschaften - gegenüber, dass ohne erweiterte Mitbestimmungsrechte und starke Einflussnahme von betrieblichen Interessenvertretungen eine arbeitswissenschaftlich fundierte und an den Kriterien menschengerechter Arbeit orientierte Gestaltung digitalisierter Arbeitsplätze nur in geringem Umfang stattfinden werde (Anlauft, Habenicht und Klippert 2019; Urban 2016; Schmidt 2017).

Im nachfolgenden Beitrag wird die Auffassung vertreten und an empirischen Beispielen zu untermauern versucht, dass technologische Innovationen in Betrieben durchaus neue Gestaltungspotenziale für eine sichere, gesunde und lernförderliche Arbeit mit sich bringen können. Zur ErschlieBung dieser Gestaltungspotenziale ist es jedoch notwendig, dass die von Transformationsprozessen betroffenen betrieblichen Akteure kollektiv befähigt und frühzeitig am Gestaltungsprozess beteiligt werden. Dabei 
kommt dem Erwerb und der Nutzung von Arbeitsgestaltungskompetenz der am Veränderungsprozess Beteiligten betrieblichen Akteure wie technisch Planenden und Arbeitsschutzakteuren, Führungskräften, Interessenvertretungen und Beschäftigten eine bedeutsame Rolle zu. Im Beitrag wird mit dem SePIAR-Verfahren ein Vorgehensmodell vorgestellt, mit dem im Rahmen von Digitalisierungsprozessen auf lokaler Ebene Arbeitsgestaltungskompetenz von Führungskräften und ihren Teams zur Gesundheitsprävention aufgebaut werden kann. SePIAR steht für ein stufenweises Vorgehen, das aus einer Stress-Sensibilisierung und dem Aufbau arbeitswissenschaftlich fundierten Gestaltungswissens (Se), einer Priorisierung relevanter Problemschwerpunkte (P), der Initiierung von Gestaltungsideen (I), der Aktivierung von Führungskräften und Mitarbeitenden zur Umsetzung (A) und der gemeinsamen Festlegung vom Umsetzungsmaßnahmen sowie einer Reflexion des Gesamtteams (R) besteht.

Der Beitrag gliedert sich wie folgt: im nächsten Abschnitt wird dargestellt, welche Präventionsstrategien derzeit in deutschen Unternehmen vorherrschen und es wird hinterfragt, inwiefern diese geeignet sind, den neuen Herausforderungen digitalisierter Arbeit gerecht zu werden. Dazu werden empirische Befunde zur Verbreitung und Qualität von Präventionsmaßnahmen sowie vorliegende arbeitswissenschaftliche Erkenntnisse über gesundheitliche Risikofaktoren von digitalisierter Arbeit herangezogen. Abschnitt 3 widmet sich der methodischen Herangehensweise, in Abschnitt 4 werden wiederum die empirischen Befunde zu Gestaltungchancen und realen Präventionshandeln bei der Einführung von Digitaltechnik am Beispiel von drei Gestaltungsprojekten in der Produktion vorgestellt. Der letzte Abschnitt beinhaltet neben einer Zusammenfassung der Ergebnisse Implikationen der Befunde für eine proaktive Gestaltung digitaler Produktionsarbeit.

\section{Prävention im Betrieb: eine Bestandsaufnahme}

Grundsätzlich bestehen in Deutschland gute institutionelle und rechtliche Rahmenbedingungen für eine präventive, d.h. Belastungen reduzierende und Ressourcen stärkende Gestaltung von Arbeit. Nach dem Arbeitsschutzgesetz ( $\$ 5$ ArbSchG) müssen physikalische, aber auch psychische Belastungen am Arbeitsplatz durch den Arbeitgeber erfasst und entsprechende Maßnahmen zur Verminderung gesundheitsschädigender Belastungen eingeleitet werden. Diese sind in regelmäßigen Abständen zu erfolgen, aber auch bei organisationalen und technischen Veränderungen, weil sich hier neue Belastungskonstellationen ergeben können (Faber und Satzer 
2016). Der Arbeitgeber ist darüber hinaus verpflichtet, entsprechend der Gefährdungen im Betrieb und der Betriebsgröße ein adäquates Arbeitsschutzmanagement aufzubauen. Hierzu existieren beispielsweise auch Regelungen zu Mindesteinsatzzeiten von Fachkräften für Arbeitssicherheit und Arbeitsmediziner*innen. Berufsgenossenschaften bzw. staatlichen Ämtern für Arbeitsschutz obliegt die Überwachung der Einhaltung der gesetzlichen Arbeitsschutznormen. Sie unterstützen Unternehmen aber auch bei dieser Aufgabe, indem sie Beratungsangebote und Erhebungsinstrumente, beispielsweise zur Identifikation von physikalischen und psychischen Gefährdungen, zur Verfügung stellen.

Immer mehr Unternehmen richten auf freiwilliger Basis auch ein betriebliches Gesundheitsmanagement ein, um präventives Handeln in Ihrem Unternehmen zu fördern. Laut einer Studie der Bundesanstalt für Arbeitsschutz und Arbeitsmedizin stieg die Anzahl von Beschäftigten, die über eine betriebliche Gesundheitsförderung in ihrem Betrieb berichten, zwischen 2006 und 2018 von 34,3 auf 44,7 Prozent (Lück et al. 2019). Diesen zunächst einmal erfreulichen Befunden steht allerdings die irritierende Tatsache gegenüber, dass in den letzten zehn Jahren die Arbeitsunfähigkeitsquoten in den Betrieben um 30 Prozent gestiegen sind (Hammer et al. 2019). Nach Schätzung der Bundesanstalt für Arbeitsschutz und Arbeitsmedizin ergibt sich für das Jahr 2018 hieraus für Betriebe ein Verlust an Bruttowertschöpfung in Höhe von insgesamt 133 Mrd. Ein Tag Arbeitsunfähigkeit verursacht hierbei je nach Branche Produktionsausfallkosten von 62 Euro bis 158 Euro (BAuA 2020).

Gesundheitsexperten machen für diese Negativentwicklung, neben demografischen Faktoren und einer Zunahme psychischer Fehlbelastungen, vor allem ineffiziente Präventionsstrategien in Unternehmen aus: viele Betriebe setzen zur Stärkung der Gesundheit ihrer Beschäftigten primär auf verhaltenspräventive Maßnahmen wie Raucherentwöhnung, Stressmanagement-Trainings oder Ernährungsprogramme (Lenhardt 2017).

Krankenkassen gaben für diese Präventionsdienstleistungen im Betrieb im Jahr 2016 6,4 Mrd. Euro aus (Hammer et al. 2019). Demgegenüber führen deutlich weniger Betriebe verhältnisbezogene Präventionsmaßnahmen durch, die auf eine systematische Gestaltung von gesundheits- und lernförderlichen Arbeitssystemen abzielen (Lenhardt 2017). Hierunter können beispielsweise Maßnahmen zur Ergonomie und alternsgerechten Arbeitsgestaltung, Arbeitszeitflexibilisierung, arbeitsschutzspezifische Qualifizierungsmaßnahmen, Gesundheitszirkel oder Gefährdungsbeurteilungen gerechnet werden (Schempp und Strippel 2017). Die Bevorzugung von eher verhaltensbezogenen Präventionsmaßnahmen im Rahmen des betrieblichen Gesundheitsmanagements erweisen sich als besonders kritisch, da 
Evaluationsstudien immer wieder die geringe Nachhaltigkeit verhaltenspräventiver Präventionsmaßnahmen nachweisen und Arbeitswissenschaftler*innen deshalb eine verstärkte Ausrichtung des betrieblichen Arbeits- und Gesundheitsschutzes auf kombinierte verhaltens- und verhältnispräventive Maßnahmen einfordern (zusammenfassend Bamberg und Busch 2006; Lenhardt 2017).

\subsection{Chancen und Risiken gesunder Arbeit durch Digitalisierung}

Die vorangegangenen Befunde zum betrieblichen Präventionshandeln leiten zu der Frage, welche möglicherweise neuen Präventionsbedarfe sich durch Digitalisierungsprozesse in Unternehmen ergeben. Die arbeitswissenschaftliche Forschung hat in den letzten Jahren zahlreiche Befunde zu potenziellen gesundheitlichen Folgewirkungen der digitalen Durchdringung unserer Arbeit zusammengetragen. Eine weitgehende Übereinstimmung der Einschätzungen sowohl von Forschenden, als auch von Personalverantwortlichen und von Interessenvertretungen besteht darin, dass durch die Digitalisierung insbesondere die psychischen Anforderungen und damit das Risiko psychischer Fehlbelastungen zunehmen können (u.a. Rothe et al. 2017; Lechleiter, Purbs und Sonntag 2019; Ahlers 2018; Haipeter 2019). In der arbeitswissenschaftlichen Forschung sind in den letzten Jahren spezifische Fehlbelastungsformen identifiziert worden, die in digitalisierten Arbeitssystemen vermehrt auftreten. Hierzu zählen:

- Überwachung: Die in Produktion und Logistik vermehrt eingesetzten Assistenzsysteme ermöglichen zum Teil die Ermittlung personenbezogener Daten (z.B. Bewegungsdaten). Meta-Analysen zeigen hier, dass der Einsatz dieser Systeme Stresserleben, eine Verminderung des Commitments und psychosomatische Erkrankungen nach sich ziehen können, wenn von Seiten des Arbeitgebers gegenüber den Beschäftigten keine Transparenz über die Datenermittlung gegeben wurde und keine Regelungen zur Vermeidung eines personenbezogenen Monitorings getroffen wurden (zusammenfassend Backhaus 2018).

- Zeitdruck- und Beschleunigungserleben: Prozessoptimierungen durch den Einsatz digitaler Werkzeuge beschleunigen Arbeitsprozesse, was zu Zeitdruckerleben und Überlastung führen kann (Trägner 2006).

- Informationsüberflutung: Die Praxis, verschiedene digitale Werkzeuge und Systeme parallel zu bearbeiten, begünstigt das Auftreten von mentalen Überforderungen (sogenannte Informationsüberflutung oder Informationsrauschen (Junghanns und Kersten 2019). Diese Form der 
Überforderung der mentalen Verarbeitungskapazität kann zu Schlafstörungen, Nicht-abschalten-können und chronischen Stressreaktionen führen (Junghanns und Kersten 2019).

- Unbeherrschbarkeit technischer Systeme: Insbesondere im Bereich der Wissensarbeit werden durch die Zunahme hoch komplexer Arbeitsprozesse und deren Abbildungen in ITK-Systemen vermehrte Belastungen durch qualitative Überforderungen erwartet (sogenannte Techno-Complexity (Gimpel, Lanzl, Manner-Romberg und Nüske 2018)). Das Gefühl einer mangelnden Beherrschbarkeit von komplexen bzw. sich selbststeuernden Systemen erhöht das Risiko von Handlungsfehlern, was bei Verantwortlichen und Maschinenoperateuren zu Stressreaktionen, Schlafstörungen und Angstzuständen führen kann (Grote 2018).

- Technologischer Anpassungsdruck: Durch den vermehrten Einsatz digitaler Werkzeuge steigt insgesamt auch der Druck zum Erwerb technologischer Methodenkompetenzen. Dies kann zu Stressreaktionen führen, wenn das Lernen als Zwang erlebt wird und die Beschäftigten keine ausreichenden Zeitressourcen zur Verfügung gestellt bekommen, sich angemessen in neue Systeme oder Systemupdates einzuarbeiten. Diese Aneignungsbehinderungen bei der Einarbeitung führen oft zu überlangen Arbeitszeiten, stehen aber auch im Zusammenhang mit psychosomatischen Erkrankungen und Erschöpfungserleben (Gerlmaier und Latniak 2013).

- Omnipräsenz-Erwartungen: Seit längerem werden auch die gesundheitlichen Auswirkungen einer zunehmenden Entgrenzung von Arbeiten und anderen Lebenssphären infolge eines verstärkten Einsatzes mobiler Endgeräte untersucht. Hier zeigt sich, dass durch die Nutzung der mobilen Endgeräte erweiterte Erreichbarkeitserwartungen und Verlängerungen der Arbeitszeiten mit sich bringen, was Regenerationsprobleme, psychische Erschöpfung und Depressivität begünstigt (u.a. Derks, ten Brummelhuis, Zecic und Bakker 2012; Dettmers et al. 2012).

- Technische Systemunzuverlässigkeit: In zunehmend mehr Studien zeigt sich auch, dass digitale Werkzeuge aufgrund ihrer unzureichenden Anpassung an das Nutzerverhalten (mangelnde „accessibility“) sowie durch die Unzuverlässigkeit von IT-Systemen (häufige Abstürze, geringe Erwartungskonformität) (u.a. Maier, Laumer und Weinert 2015) zu erheblichen psychischen Belastungen wie Arbeitsunterbrechungen und ungeplanten Zusatzaufwand sowie zu Zeitdruck führen können. Als technisch bedingte Störquellen stellen diese Belastungen weitere Risikofaktoren für den Erhalt der psychischen Gesundheit dar (Robelski 2016; Gimpel et al. 2018). 
Durch die Digitalisierung werden aber auch erhebliche Gestaltungspotenziale für eine gesundheits- und lernförderliche Arbeit erwartet. Sie stellen Ressourcen dar, die sich günstig auf das Wohlbefinden auswirken oder gesundheitsschädigende Wirkungen neutralisieren können (Bakker und Demerouti 2007). Vermehrte Gestaltungspotenziale durch die Digitalisierung werden unter anderem in neuen Optionen für eine Takt ungebundene und damit weniger fremdbestimmten Montagesystemen ebenso gesehen wie in agiler Teamarbeit oder dem vermehrten Einsatz von Home-OfficeLösungen. Die wenigen Feldstudien zu diesen Themenbereichen verweisen aber auch darauf, dass die Gesundheitspotenziale durch den Technikeinsatz oft nur dann erschlossen werden können, wenn Kriterien menschengerechter Arbeit frühzeitig in den Neugestaltungsprozess berücksichtigt werden und den Beschäftigten hierbei weitreichende Mitentscheidungsmöglichkeiten bei der Arbeitsgestaltung zuteilwerden (u.a. Bamberg und Busch 2006; Höge und Büssing 2004; Ducki und Nguyen 2016). Betrachtet man allerdings die bisherigen Befunde zu den psycho-sozialen Auswirkungen der Digitalisierung in der Arbeitswelt, so gibt es bei Beschäftigtenbefragungen Hinweise darauf, dass von Digitalisierung Betroffene oft höhere Belastungen, aber nur selten auch höhere Arbeitsressourcen erleben: An hochgradig digitalisierten Arbeitsplätzen berichten verschiedenen Studien zufolge Beschäftigte häufiger über Zeitdruck, Überforderungen durch Informationsüberflutung, Omni-Präsenzerwartungen und einen hohen technologischen Anpassungsdruck (DGB 2017; Gimpel et al. 2018). Davon betroffene Beschäftigte wiederum haben ein deutlich erhöhtes Risiko für stressinduzierte Erkrankungen wie psychische Erschöpfung, Schlafstörungen, Kopfschmerzen oder Verspannungen (Böhm et al. 2016).

\subsection{Gelingensfaktoren für die präventive Gestaltung digitaler Arbeitssysteme: das Konzept der Organisationalen Gestaltungskompetenz}

Die zuvor beschriebenen Befunde können durchaus Anlass zu der Einschätzung geben, dass der institutionalisierte betriebliche Arbeits- und Gesundheitsschutz angesichts von Herausforderungen wie der Globalisierung und Digitalisierung an seine Grenzen stößt. Mit seinem Regelungsgerüst scheint dieser vor allem wenig erfolgreich darin zu sein, insbesondere die steigende Anzahl psycho-sozialer Belastungen und Erkrankungen erfolgreich einzudämmen. Dazu dürfte beitragen, dass derartige Stressrisiken im betrieblichen Gesundheitsmanagement primär verhaltenspräventiv angegangen werden, weil diese Vorgehensweise mit weniger (unerwünschten) Eingriffen in bestehende Arbeitssysteme realisiert werden können. Sub- 
jektorientierte betriebliche Präventionskonzepte fußen auf der Annahme, dass Beschäftigte steigende Anforderungen durch die Stärkung ihres Bewältigungsverhaltens am besten bewältigen können. Sie verweisen häufig auch darauf, dass in flexiblen Arbeitssystemen mit ihrer hohen Dynamik immer weniger Gestaltungsfreiräume bestehen, um diese Arbeitssysteme nach den Kriterien menschengerechter Arbeit zu gestalten (Janneck und Hoppe 2018; Weiß 2012).

Diesen eher gestaltungspessimistischen Perspektiven gegenüber stehen arbeitspolitische Initiativen insbesondere von Gewerkschaften. Diese verfolgen das Ziel, durch den aktuellen Digitalisierungsdiskurs Wendungen in Richtung einer nachhaltigen betrieblichen Arbeitspolitik und -regulierung zu forcieren. Ein neues Leitbild nachhaltiger Arbeit besteht hier unter anderem darin, Digitalisierungsprozesse in Unternehmen proaktiv zu gestalten. Unter einer proaktiven Arbeitsgestaltung wird dabei ein betriebliches Gestaltungshandeln verstanden, dass auf eine frühzeitige Einflussnahme von Beschäftigten und ihren Interessenvertretungen bei betrieblichen Transformationsprozessen setzt und die Sicherung von Beschäftigung, Stärkung von Gesundheitsressourcen, Qualifizierung und Wahrung von Persönlichkeitsrechten zum Ziel hat (Anlauft et al. 2019). Dies führt zu der Frage, inwieweit eine solche proaktive Arbeitsgestaltung bei Digitalisierungsprozessen heute in Betrieben stattfindet und welche Gelingensfaktoren es für eine solche proaktive Gestaltung bedarf.

Im Rahmen des vom BMBF-geförderten Verbundvorhabens „Initiative betriebliche Gestaltungskompetenz stärken - ein Modellvorhaben für Unternehmen und Beschäftigte der Elektro- und Metallindustrie" (InGeMo) ${ }^{1}$ wurde davon ausgegangen, dass ein wesentlicher Gelingensfaktor für eine proaktive Arbeitsgestaltung in der Förderung von kollektiver Arbeitsgestaltungskompetenz und der Förderung arbeitsimmanenter Lernprozesse zu sehen ist (Gerlmaier 2018a; Gerlmaier 2018b).

Organisationale Gestaltungskompetenz wird in diesem Zusammenhang als eine kollektive Fähigkeit verstanden, Arbeitssysteme so zu gestalten, dass sie psycho-soziale Gesundheitsressourcen stärken und psycho-physiologische Risiken vermindern (Gerlmaier 2018a). Dies umfasst die kollektive Fähigkeit der im Unternehmen Agierenden, gesundheitswidrige Systemzustände zu erkennen, und entsprechend ihrer individuellen Hand-

1 Das Verbundprojekt „Initiative betriebliche Gestaltungskompetenz stärken - ein neues Präventionsmodell für Unternehmen und Beschäftigte“ (InGeMo) wurde gefördert vom Bundesministerium für Bildung und Forschung (BMBF), Förderkennzeichen 02L14A020, Laufzeit von April 2016 bis Juli 2019. 
lungsvoraussetzungen Ressourcen zu mobilisieren, um psychische Gesundheit und Beschäftigungsfähigkeit wiederherzustellen und weiterzuentwickeln. Konzeptionell umfasst der Begriff der Gestaltungskompetenz Motivations-, Wissens- und Handlungsregulationsaspekte (vgl. Erpenbeck und von Rosenstiel 2007). Dazu werden die Komponenten „stressbezogenes Gefahrenwissen“, „arbeitsbezogenes Gestaltungswissen“, „arbeitsbezogene Handlungskompetenz" sowie "Gestaltungsmotivation" gerechnet (siehe Tabelle 1). Proaktive Arbeitsgestaltung kann diesem Konzept zufolge nur dann zu erfolgreichen Präventionsergebnissen führen, wenn neben einer umfangreichen Beteiligung auch ein ausreichendes Ausmaß kollektiver Gestaltungskompetenz bei den am Gestaltungsprozess beteiligten Beschäftigten, Führungskräften, Interessenvertretungen und Arbeitsschutzexpert*innen besteht.

Digitale Transformationsprojekte in Betrieben können demzufolge schnell zu negativen gesundheitlichen Folgewirkungen führen, wenn kaum substanzielles Gefahrenbewusstsein (etwa für die Wirkungen von Stress) bei den Gestaltenden vorhanden ist und sich die Gestaltungsakteure in Sachen Arbeitsgestaltung nur auf ihre Intuition verlassen. Auch die in einer Organisation möglicherweise vorhandenen Regeln, Regulierungsoptionen oder Mitwirkungsmöglichkeiten kommen für eine proaktive Arbeitsgestaltung dann kaum zum Tragen, wenn es den betrieblichen Akteuren an Arbeitsgestaltungskompetenz und Gestaltungsmotivation fehlt, Ansprüche an menschengerechte Arbeit geltend zu machen und diese auch durchzusetzen. Und letztlich können sich in Organisationen nur dann flächendeckend kollektive Präventionskulturen durchsetzen, wenn Beschäftigten und ihren Führungskräften Lern- und Reflexionsräume eingeräumt werden, um Arbeitsgestaltungskompetenz für ihre Tätigkeiten zu erwerben und diese in ihren Arbeitsbereichen umsetzen zu können. Organisationale Gestaltungskompetenz stellt somit eine wichtige Stellgröße dar, um Prozesse einer proaktiven Arbeitsgestaltung in Unternehmen zu etablieren. Sie ermöglicht es, betrieblichen Entscheidern und Beschäftigten, sich kompetent an der Gestaltung des Arbeitssystems zu beteiligen und Gestaltungspotenziale für eine gesundheits- und lernförderliche Arbeit zu erschließen. 
Tabelle 1: Wirkebenen und Indikatoren organisationaler Gestaltungskompetenz

\begin{tabular}{|l|l|}
\hline Zielgröße & Indikator \\
\hline $\begin{array}{l}\text { Stressbezogenes } \\
\text { Gefahrenwissen }\end{array}$ & $\bullet \begin{array}{l}\text { Kenntnisse über die Risiken von Stress und Fehl- } \\
\text { belastungen }\end{array}$ \\
\hline $\begin{array}{l}\text { Arbeitsbezogenes } \\
\text { Gestaltungswissen }\end{array}$ & $\bullet \begin{array}{l}\text { Kenntnisse über Strategien zur Stressprävention bzw. } \\
\text { Belastungsverminderung }\end{array}$ \\
\hline Gestaltungsmotivation & $\bullet \begin{array}{l}\text { Interesse an der Minderung von Stressfolgen und dem } \\
\text { Aufbau arbeitsbezogener Ressourcen. }\end{array}$ \\
\hline $\begin{array}{l}\text { Interesse an der Entwicklung von Gestaltungsmaßnah- } \\
\text { men zur Stressreduzierung und der Förderung von } \\
\text { Gesundheit }\end{array}$ \\
Arbeitsbezogene & $\bullet \begin{array}{l}\text { Nutzung stressreduzierender Bewältigungsstrategien } \\
\text { (z. B. Durchführung von Kurzpausen oder Blockzeiten für } \\
\text { konzentriertes Arbeiten) }\end{array}$ \\
$\begin{array}{l}\text { Initiierung von bzw. Mitwirkung bei stresspräventiven } \\
\text { Maßnahmen im jeweiligen Handlungs- bzw. Verantwor- } \\
\text { tungsbereich (z. B. im Rahmen von Steuerungskreisen } \\
\text { oder Entscheidungsgremien) }\end{array}$ \\
\hline
\end{tabular}

Quelle: Eigene Darstellung

\subsection{Fragestellungen}

Im Rahmen der nachfolgenden empirischen Fallstudien wird im Wesentlichen zwei Fragestellungen nachgegangen: zum einen wird für verschiedene Tätigkeitsbereiche der Produktion betrachtet, inwieweit sich proaktive und präventive Arbeitsgestaltung bei der Einführung verschiedener Formen von Digitaltechnik beobachten lassen und welche Auswirkungen dies aus der Sicht von betroffenen Führungskräften und Beschäftigten auf die Ressourcen- und Gesundheitssituation hat. Zum anderen wird auf Basis eines Interventionsstudien-Ansatzes betrachtet, inwiefern Teams und ihre Führungskräfte durch den Aufbau von Arbeitsgestaltungskompetenz befähigt werden können, präventive Gestaltungspotenziale durch technische Reorganisationen innerhalb ihres Arbeitsbereiches zur Gesundheitsstärkung zu erschließen und wo hier die Grenzen der Arbeitsgestaltung liegen.

\section{Methodisches Vorgehen}

Im Rahmen des Forschungsprojektes InGeMo bestand ein zentrales Forschungsziel darin, partizipationsorientierte Konzepte zur Förderung kol- 
lektiver Arbeitsgestaltungskompetenz zu entwickeln, diese in Unternehmen zu erproben und ihre Wirksamkeit zu überprüfen. Dabei wurde mit Methoden der Aktionsforschung gearbeitet. Ziele der Aktionsforschung bestehen in erster Linie in der Lösung lokaler praktischer Probleme von Organisationen oder Organisationseinheiten unter starken Einbezug der Betroffenen. Hierzu werden die jeweiligen Kontexte gemeinsam von Forscher*innen und Praktiker*innen beschrieben und Theorien zu möglichen Problemursachen gebildet und daraus abgeleitete Interventionen entwickelt. Diese sollen dann durch Interventionsexperimente getestet werden (Richenhagen und Dick 2019). Entsprechend eines Phasenmodells wurden in den untersuchten Fallbereichen Phasen der Planung, Handlung, Reflektion und Evaluierung durchlaufen (Dickens und Watkins 1999).

In der Planungsphase erfolgten in den ausgewählten Fallbereichen zunächst Betriebsbegehungen und anschließende Interviews mit zuständigen Führungskräften, Betriebsrät*innen und Arbeitsschutz-Verantwortlichen. Hierbei wurden betriebliche Kontextfaktoren des jeweiligen sozio-technischen Arbeitssystems ebenso erfasst wie Belastungen und Gestaltungspotenziale, die sich aus den jeweiligen technischen Reorganisationen ergaben. Diese Daten wurden ergänzt durch abteilungsspezifische schriftliche Mitarbeiterbefragungen (Vollerhebungen), in denen ebenfalls Belastungsund Ressourcenanalysen vor Beginn der Gestaltungsprojekte und sechs Monate später vorgenommen wurden. In getrennten Mitarbeiter- und Führungskräfte-Workshops erfolgte im Anschluss daran zunächst eine Stress-Sensibilisierung, Priorisierung von Handlungsbedarfen und ein Aufbau entsprechenden Gestaltungswissens, zum Beispiel zur Reduzierung von Arbeitsbelastungen oder der Förderung von Erholung (Gerlmaier 2020). Im Rahmen der Workshops wurden von den Führungskräften und Mitarbeitenden zunächst getrennt Gestaltungsmaßnahmen entwickelt und nach einer kollektiven Reflexionsphase des Teams und seiner Führungskräfte umgesetzt.

Sechs bis acht Monate nach der Umsetzung der Maßnahmen erfolgte wiederum durch das Forschungsteam eine Evaluation der Umsetzungsmaßnahmen im Hinblick auf Gesundheitsparameter und Veränderungen der Belastungs- und Ressourcensituation. An den Führungskräfte-Workshops nahmen nach Möglichkeit Führungskräfte aller Ebenen des Arbeitsbereichs (Abteilungsleitung, Meister, Vorarbeiter) teil sowie Beschäftigte aus der Früh- und Mittagsschicht. 


\subsection{Erhebungsinstrumente}

Zur Beantwortung der im Beitrag aufgeworfenen Fragen wurden Interviews mit für die Abteilung zuständigen Führungskräften, Betriebsräten und Arbeitsschutz-Akteuren aus der Planungsphase sowie die Sitzungsdokumentationen aus der Reflexionsphase herangezogen. Veränderungen bei der Gestaltungskompetenz, psycho-sozialen Gesundheit, Arbeitsintensität sowie bei den verschiedenen berücksichtigten arbeitsbezogenen Ressourcen erfolgten über ein schriftliches Befragungsinstrument, das vor Beginn und sechs bis acht Monate nach Abschluss der Maßnahmen von den Führungskräften und Mitarbeitenden der Fallbereiche ausgefüllt wurde. Skalen und Beispiel Items finden sich in Tabelle 2.

\section{Tabelle 2: Skalen und Beispiel-Items des Befragungsinstruments}

\begin{tabular}{|l|c|l|}
\hline Skala & $\begin{array}{c}\text { Anzahl } \\
\text { Items }\end{array}$ & Beispiel-Item \\
\hline $\begin{array}{l}\text { Psychische } \\
\text { Gesundheit }\end{array}$ & 17 & $\begin{array}{l}\text { Ich fühle mich wieder müde, wenn ich aufstehe und } \\
\text { den nächsten Arbeitstag vor mir habe. }\end{array}$ \\
\hline $\begin{array}{l}\text { Arbeitsbezogene } \\
\text { Handlungskompetenz }\end{array}$ & 5 & $\begin{array}{l}\text { Wo dies arbeitsorganisatorisch möglich ist, plane ich } \\
\text { Zeiten für störungsfreies Arbeiten ein und nehme sie } \\
\text { mir dann auch. }\end{array}$ \\
\hline Gestaltungsspielraum & 5 & $\begin{array}{l}\text { Wieviel Einfluss haben Sie auf die Reihenfolge der zu } \\
\text { erledigenden Arbeitsaufgaben? }\end{array}$ \\
\hline Zeitsouveränität & 4 & $\begin{array}{l}\text { Wieviel Einfluss haben Sie bei der Planung der } \\
\text { Arbeitszeit (Schichten, Überstunden)? }\end{array}$ \\
\hline $\begin{array}{l}\text { Soziale Unterstützung } \\
\text { durch Kolleg*innen }\end{array}$ & 3 & $\begin{array}{l}\text { Wie sehr können Sie sich auf die folgenden Personen } \\
\text { verlassen, wenn in der Arbeit Probleme auftauchen? }\end{array}$ \\
\hline $\begin{array}{l}\text { Soziale Unterstützung } \\
\text { durch Vorgesetzte }\end{array}$ & 3 & $\begin{array}{l}\text { Wie sehr sind diese Personen bereit, Ihre Probleme in } \\
\text { der Arbeit anzuhören? }\end{array}$ \\
\hline $\begin{array}{l}\text { Kapazitätspuffer } \\
\text { (Polyvalenz, Redun- } \\
\text { danz) }\end{array}$ & 4 & $\begin{array}{l}\text { Die Arbeitsaufgaben können zusammen mit einem } \\
\text { oder mehreren Kollegen/ im Arbeitstandem durch- } \\
\text { geführt werden (z.B. durch gemeinsame/sich über- } \\
\text { lappende Arbeitsbereiche) }\end{array}$ \\
\hline $\begin{array}{l}\text { Qualifizierungs- } \\
\text { möglichkeiten }\end{array}$ & 2 & $\begin{array}{l}\text { Es ist möglich, sich während der Arbeit in neue Auf- } \\
\text { gaben einzuarbeiten. }\end{array}$ \\
\hline $\begin{array}{l}\text { Quantitative } \\
\text { Überforderung }\end{array}$ & 4 & $\begin{array}{l}\text { Man muss Dinge tun, für die man eigentlich zu wenig } \\
\text { ausgebildet und vorbereitet ist. }\end{array}$ \\
\hline
\end{tabular}

Quelle: Eigene Darstellung 


\subsection{Beschreibung der Untersuchungsfälle}

Zur Beantwortung der Fragen wurden aus einem Gesamtsample von acht Gestaltungsfällen drei Fälle aus unterschiedlichen Tätigkeitsbereichen der Produktion ausgewählt. Hierbei handelte es sich um einen Bereich mit angelernten Maschinenbediener"innen, mit überwiegend qualifizierten Facharbeiter"innen sowie hochqualifizierten Angestellten aus dem Bereich des technischen Projektmanagements. Die drei ausgewählten Untersuchungsfälle stellen für die Tätigkeitsbereiche im Hinblick auf die betrieblichen Kontextfaktoren, Belastungs- und Ressourcensituationen typische Fallkonstellationen dar, die vergleichbar sind mit anderen Fällen aus unserem Sample. Die Fokussierung auf diese drei Fälle wurde vorgenommen, um die zugrunde liegenden Reorganisations- und Gestaltungsprozesse in den einzelnen Arbeitsbereichen detaillierter vorstellen zu können. Bei den ausgewählten Fällen handelt es sich um zwei Bereiche aus einem Metall produzierenden und verarbeitenden Unternehmen mit ca. 4000 Beschäftigten sowie einem mittelständischen Zuliefererunternehmen der Automobilbranche mit ca. 800 Beschäftigten. Die Fallauswahl erfolgte mit dem Ziel, die Auswirkungen digitalgetriebener Reorganisationen in Tätigkeitsbereichen mit unterschiedlichen Qualifikationsniveaus zu betrachten, da Arbeitssystem bedingt unterschiedliche Gestaltungsvoraussetzungen angenommen werden müssen (Huchler 2019).

\subsubsection{Fallbeispiel 1: Adjustage}

Bei dem Untersuchungsbereich mit überwiegend angelernten Beschäftigten, nachfolgend als Fall Adjustage bezeichnet, handelt es sich um einen Arbeitsbereich aus einem Stahl produzierenden und verarbeitenden Unternehmen. Die ca. 30 Beschäftigten des Arbeitsbereiches arbeiten in einem Dreischichtsystem. Dort werden von den überwiegend angelernten Beschäftigten Metallvorprodukte an verschiedenen Betriebsaggregaten bearbeitet (z.B. Sägeeinrichtungen, Wärmebehandlung). Ein Großteil der Beschäftigten ist über 50 Jahre alt, männlich und verfügt über einen geringen Bildungsabschluss. Etwa ein Jahr vor der Untersuchung hatte nach Angaben des Abteilungsleiters im Untersuchungsbereich aufgrund eines Nachfrageeinbruchs ein deutlicher Personalabbau stattgefunden, der für die Beschäftigten zu einer Aufgabenanreicherung um logistische Aufgaben (Beund Entladen, Intralogistik) führte. Ungefähr sechs Monate nach dieser Reorganisationsmaßnahme wurde darüber hinaus durch den Abteilungsleiter ein digitalgestütztes Produktionsplanungstool mit dem Ziel einge- 
führt, einen besseren Überblick über die Produktionskosten einzelner Vorproduktetypen zu erhalten und den Personaleinsatz effizienter zu gestalten. Dazu sind zusätzliche PC-Stationen an den analogen Maschinen eingerichtet worden, an denen die Maschinenoperateure nun zusätzlich manuell Daten zur Bearbeitungsdauer der Werkstücke und Störungszeiten eingeben müssen.

\subsubsection{Fallbeispiel 2: Werkzeng}

Der ausgewählte Fall Werkzeug stellt einen Arbeitsbereich mit 34 Beschäftigten dar, in dem Werkzeuge und Bauteile für die Automobilindustrie in Einzelanfertigung hergestellt werden. Im Bereich arbeiten bis auf zwei Frauen mehrheitlich Männer mit einer Facharbeiterausbildung zur Werkzeugmacher*in, Programmierer*in oder Schlosser*in. Der Altersdurchschnitt ist mit 45 Jahren etwas geringer als im Fall Adjustage. Im Fall Werkzeug arbeiten mehrere Teilteams, bestehend aus Auftragsannahme, Arbeitsvorbereitung, Programmierung, Werkzeugausgabe und Maschinenbedienung innerhalb einer Fertigungskette zusammen. In der Produktion sind die Beschäftigten im Dreischichtsystem tätig, die Beschäftigten in der Programmierung und Auftragsannahme arbeiten dagegen nur in der Tagschicht. Die Abteilung wurde in das Untersuchungssample aufgenommen, weil ca. zwölf Monate vor der Untersuchung ein neues digital gestütztes System zur Prozesssteuerung (Manufacturing Execution System, MES) eingeführt wurde. ME-Systeme übernehmen Anteile der Prozesssteuerung innerhalb eines Herstellungsprozesses und zielen darauf ab, Arbeitsprozesse an der Maschine zu standardisieren und damit unter anderem Materialkosten zu reduzieren.

\subsubsection{Fallbeispiel 3: Projektmanagement}

Der dritte Untersuchungsfall stammt aus einem mittelständischen Zuliefererbetrieb für die Automobilindustrie. Stellvertretend für den Tätigkeitsbereich der produktionsnahen Wissensarbeit wird hier der Arbeitsbereich Projektmanagement betrachtet, indem für die Angebotserstellung vorgelagerte Wirtschaftlichkeitsberechnungen und Analysen zur technischen Machbarkeit vorgenommen werden. Im beschriebenen Arbeitsbereich sind ca. 45 Mitarbeitende in sechs Teilteams für die Angebotserstellung spezifischer Automobilhersteller zuständig. Im Team arbeiten überwie- 
gend Ingenieure*innen und Fachwirt*innen für Betriebswirtschaft, die mehrheitlich männlichen Teammitglieder (14 Prozent Frauenanteil) bearbeiten in der Regel mehrere Kundenprojekte parallel. Das Projektmanagement im Fallbereich ist durch ein relativ hohes Ausmaß an Selbstorganisation gekennzeichnet; viele Teammitglieder führen Verhandlungen mit Verantwortlichen der beauftragenden Automobilhersteller eigenverantwortlich durch. Im Gegensatz zu den zuvor beschriebenen Fällen aus der Produktion liegen für die Projektmitarbeitenden flexible Gleitzeitregelungen und Möglichkeiten zum Home-Office vor. Der Fall wurde ausgewählt, weil hier ein für produktionsnahe Wissensarbeit typischer sehr hoher Nutzungsgrad an unterschiedlichen ITK-Systemen vorzufinden ist: die Tätigkeiten erfordern Methodenkompetenz zur Nutzung unter anderem von ERP- und CAD-Anwendungen, Projektmanagement-Tools und Officeanwendungen. Da das untersuchte Unternehmen Teil des Produktionsnetzwerks verschiedener Automobilkonzerne darstellt, sind die genutzten ITInfrastrukturen nach Darstellung des befragten Abteilungsleiters vollständig durch die Herstellerkonzerne vorgegeben, so dass hierdurch das Zuliefererunternehmen kaum Gestaltungsspielräume bei der Wahl der unternehmensspezifischen ITK-Infrastruktur besaß.

\section{Ergebnisse}

\subsection{Präventives und proaktives Gestaltungshandeln bei der Einführung von Digitaltechnik}

Im ersten Schritt der Analyse sollte der Frage nachgegangen werden, inwieweit sich in den drei Fallbereichen bei der Einführung neuer Digitaltechnik betriebliche Praktiken proaktiver bzw. präventiver Arbeitsgestaltung beobachten lassen. Hierzu wurden im Rahmen des Forschungsprojektes in der Phase der Problemfindung mit den zuständigen Führungskräften und ihren Teammitgliedern auf Basis ihrer Belastungsdaten in getrennten Workshops diskutiert, inwiefern bei der jeweiligen Einführung von Digitaltechnik frühzeitig Aspekte von Gesundheitsschutz, Qualifizierung, Beschäftigungserhalt und Wahrung persönlicher Schutzrechte durch die Gestaltenden berücksichtigt wurden und inwiefern sich hieraus neue Belastungen oder neue Freiheitsgrade ergeben hatten. Die Führungskräfte aus den drei Fallbereichen berichteten hierbei übereinstimmend, dass sie ihre Beschäftigten bei der Einführung der Digitaltechnik frühzeitig über die Maßnahme, ihre Ziele und mögliche Auswirkungen auf die Beschäftigung sowie Zeithorizonte bei der Umsetzung informiert hatten. Lediglich 
im Fallbereich Werkzeug erhielten ausgewählte Beschäftigte darüber hinaus die Möglichkeit, an der Auswahl der neuen Technik (hier: MES) mitzuwirken, indem sie an den Vorführungen der verschiedenen Produktanbieter beteiligt wurden. Eine technisch durchaus mögliche Mitwirkung von Teammitgliedern an der Entwicklung des Produktionsplanungstools im Fallbereich Adjustage wurde durch den Abteilungsleiter nicht vorgesehen, da dieser das Kompetenzniveau der Beschäftigten als zu gering ansah. Im Bereich Projektmanagement wiederum bestand aus Sicht des Abteilungsleiters keine Einflussmöglichkeit aufgrund der Vorgaben der Kund*innen für bestimmte Softwareprodukte. Auch im Bereich der vorausschauenden Qualifizierung zeigte sich in den drei Fallbereichen wenig proaktives Gestaltungshandeln durch die Führungskräfte: keiner nutzte die Einführung der Digitaltechnik, um betroffene Beschäftigte für neue Tätigkeiten höher zu qualifizieren (Upgrading, vergleiche Hirsch-Kreinsen 2017). Lediglich im Bereich Werkzeug erhielten alle Beschäftigten mehrtägige Schulungen zum Umgang mit dem ME-System. Im Bereich Adjustage erfolgten nur kurze Einweisungen am Arbeitsplatz durch die Vorarbeiter in die Nutzung des Planungstools. Im Projektmanagement stellte die Abteilungsleitung den Beschäftigten bei der Einführung neuer digitaler Werkzeuge in der Regel entsprechenden technischen Support über Hotlines der Produkthersteller und Produktdokumentationen zur Verfügung und erwartete, dass sich die Projektmitarbeitenden eigenständig in die neue Technik einarbeiteten. Im Planungs- und Einführungsprozess sahen Führungskräfte in allen drei Fallbereichen auch keine Notwendigkeit, prospektive Gefährdungsbeurteilungen zur frühzeitigen Erkennung neuartiger psychischer oder physikalischer Belastungen einzuleiten, obwohl dies durch den Gesetzgeber vorgeschrieben ist. Vielmehr waren insbesondere die Bereichsleitungen der Meinung, dass es ausreiche, aus dem Technikeinsatz auftretende Belastungen zu bearbeiten, wenn die Beschäftigten hierüber ihren Unmut äußerten. Keinerlei Bedarf für proaktives Gestaltungshandeln sahen die Führungskräfte auch bei Aspekten der Wahrung persönlicher Schutzrechte für den Fall von technischen Überwachungsmöglichkeiten durch das System. Im Fallbeispiel Adjustage räumte der Abteilungsleiter zwar ein, dass mithilfe des Produktionsplanungstools überwachbar sei, welche Teammitglieder häufiger aus seiner Sicht falsche Dateneingaben tätigten. Er sah aber keine Notwendigkeit, hierzu Regelungen mit dem zuständigen Betriebsrat zu treffen, solange dieser ihn hierzu nicht auffordere.

Im Rahmen der Mitarbeiter"innen-Workshops wiederum wurde deutlich, dass viele Workshop-Teilnehmer*innen die wenig vorausschauende Einführung der Digitaltechnik als Ursache für eine weitere Zunahme ihres Stresserlebens und gesundheitlicher Probleme ansahen. Häufig äußerten 
die Mitarbeitenden im Workshop auch, dass sie sich aufgrund der geringen Mitwirkungsmöglichkeiten und der vom Management oft kommunizierten Sachzwanglogik den technischen Veränderungen eher ausgeliefert fühlten, als dass sie hierin substanzielle Spielräume für eine Verbesserung der Arbeitsqualität erkennen konnten:

Im Fallbeispiel Adjustage machten die Teilnehmer*innen des Mitarbeiterworkshops das Produktionsplanungstool für eine weitere Intensivierung ihrer ohnehin schon hohen Arbeitsbelastungen verantwortlich. Die Mehrheit berichtete über neue Angstgefühle, dass Planungstool falsch zu bedienen und hierdurch Sanktionen zu erfahren. Die neuen Visualisierungsmöglichkeiten der Durchlaufzeiten erlebten viele der Maschinenbediener als unangemessene Überwachungsmaßnahme der Arbeitsleistung. Um nicht in den kritischen Bereich der Soll-Laufzeiten zu kommen, steigerten nach eigenen Angaben einige Maschinenbediener ihr Arbeitstempo, was wiederum zu einer Zunahme körperlicher Belastung und entsprechender Beschwerden wie Rückenschmerzen führte. Zur Verschlechterung des Betriebsklimas trug aus Sicht der Mitarbeitenden auch bei, dass die Bereichsleitung am Wochenende häufiger Revisionen der Soll-Zeiten vornahm, was von den Beschäftigten als willkürlich erlebt wurde.

Auch im Fallbeispiel Werkzeug berichteten die Beschäftigten in den Workshops mehrheitlich, dass sich nach der Einführung des MES eher Arbeitsbelastungen denn neue Gestaltungsmöglichkeiten ergeben hätten. Zwar waren die Beschäftigten mit den Qualifizierungsmaßnahmen für die Nutzung des Systems weitgehend zufrieden. Als kritisch wurde aber angesehen, dass sich durch das MES erhebliche Funktionseinschränkungen und damit Autonomieverluste für die Maschinenbediener*innen ergaben: Durch das MES entfiel die zuvor geistig anspruchsvolle 2,5-D-Programmierung durch die Maschinenoperateure, da diese nun durch die Programmierung vorgegeben war. So nahm das MES nunmehr auch die Auswahl der Fräswerkzeuge vor, was zu erheblichen Einschränkungen der Eingriffsmöglichkeiten an der Maschine führte. Die neue Funktionsteilung verursachte wiederum im Programmierteam Überlastungen und Zeitdruck. Alle Teammitglieder klagten auch über neue Entgrenzungsprobleme, weil die Kolleg*innen aus der Spät- bzw. Nachtschicht sie aufgrund der neuen Eingriffseinschränkungen häufiger zu Hause telefonisch kontaktierten. Übereinstimmend berichteten die Führungskräfte und Mitarbeitenden, dass nach der MES-Einführung zwischen den Teilgruppen und den Vorgesetzten erhebliche soziale Spannungen aufgrund neuer Arbeitsteilungen entstanden waren. Von den Führungskräften wurde hierbei beklagt, dass sie sich für einen produktiven Umgang mit diesen Konflikten nicht ausreichend qualifiziert sahen. Die Teilnehmenden des Führungskräfte- und des 
Mitarbeitenden-Workshops äußerten übereinstimmend ein starkes Bedürfnis, die bestehenden Probleme zu lösen.

Auch im Fall Projektmanagement bewerteten die Workshop-Teilnehmer*innen die Einführung neuer digitaler Technologien als häufige Quelle von Stress: unzureichende Einarbeitungszeiten und -formate führen hier immer wieder zu dem Gefühl der Unbeherrschbarkeit der Systeme. Durch Systemabstürze, automatische Software-Updates oder einer unergonomischen Benutzerführung kommt es immer wieder zu ungeplanten Zusatzaufwand und Zeitdruck, weil technisch bedingte Arbeitsunterbrechungen und Einarbeitungszeiten in der Personalkapazitätsplanung grundsätzlich nicht eingeplant würden. Anders als im Fall Adjustage und Werkzeug sahen die Workshop-Teilnehmer*innen die hauptsächlichen Ursachen ihres Stresses in den stetig steigenden Ergebnisvorgaben. Die IT- Infrastruktur wurde von ihnen kaum als Entlastungsmöglichkeit, sondern als Methode des Managements bewertet, noch mehr Aufträge in kürzerer Zeit abzuarbeiten. Sie berichteten, dass mögliche Chancen für eine Arbeitsentlastung, zum Beispiel Home-Office für konzentriertes Arbeiten in den Teambesprechungen kaum thematisiert worden waren, obwohl dies technisch möglich war und Betriebsvereinbarungen hierzu vorlagen. Neben einem durch die Digitalisierung bedingten steigenden Zeitdruck bewerteten die Beschäftigten aus dem Projektmanagement die hohen Geräuschpegel aufgrund von Video- und Telefonkonferenzen in den Mehrpersonenbüros als massiven Belastungsfaktor. Die lärmbedingten Konzentrationsstörungen wurden aus ihrer Sicht verstärkt durch den zunehmenden Wegfall geregelter Frühstückspausen, die aufgrund der Verfügbarkeitsansprüche der internationalen Kunden Telefon- und Videokonferenzen zum Opfer fielen. In Tabelle 3 sind die in den Workshops thematisierten digitalen Stressoren in den drei Fallbereichen dargestellt. 


\section{Tabelle 3: Von den Workshop-Teilnehmer*innen berichtete digitale Stressoren} in den drei Fallbereichen

\begin{tabular}{|l|c|c|c|}
\hline Berichtete digitale Stressoren & Adjustage & Werkzeug & $\begin{array}{l}\text { Projekt- } \\
\text { management }\end{array}$ \\
\hline Überwachung & $\mathrm{x}$ & $\mathrm{x}$ & $\mathrm{x}$ \\
\hline $\begin{array}{l}\text { Zeitdruck- und Beschleunigungs- } \\
\text { erleben }\end{array}$ & (Programmierung) & $\mathrm{x}$ & $\mathrm{x}$ \\
\hline Informationsüberflutung & $\mathrm{x}$ & $\mathrm{x}$ & $\mathrm{x}$ \\
\hline $\begin{array}{l}\text { Unbeherrschbarkeit technischer } \\
\text { Systeme }\end{array}$ & $\mathrm{x}$ & $\mathrm{x}$ & $\mathrm{x}$ \\
\hline Technologischer Anpassungsdruck & $\mathrm{x}$ & & $\mathrm{x}$ \\
\hline Omnipräsenz-Erwartungen & $\begin{array}{c}\mathrm{x} \\
\text { unzuverlässigkeit }\end{array}$ & & \\
\hline
\end{tabular}

Quelle: Eigene Darstellung

Hier wird sichtbar, dass es aus der Perspektive der Beschäftigten in allen drei Fallbereichen durch die Technikeinführung zunächst zu einer Zunahme von Belastungen kam. Während in allen drei Fällen von einer Zunahme von Zeitdruck- und Beschleunigungserleben sowie Belastungen durch einen technologischen Anpassungsdruck berichtet wurde, differenzieren sich alle anderen Formen von digitalen Stressoren offenbar tätigkeitsspezifisch aus.

\subsection{Präventives Gestaltungshandeln nach den Arbeitsgestaltungsworkshops}

Im ersten Analyseschritt hat sich bei den drei dargestellten Fällen unabhängig vom Tätigkeitsbereich gezeigt, dass gestaltungsverantwortliche Führungskräfte zurzeit offenbar kaum systematisch proaktives Gestaltungshandeln bei technischen Transformationsprozessen an den Tag legen und dies offenbar Konsequenzen für das Stresserleben der davon betroffenen Beschäftigten hat. Der zweite Analyseschritt im Rahmen des Beitrags widmet sich der Frage, ob durch den Aufbau von kollektiver Gestaltungskompetenz bisher ungenutzte präventive Gestaltungspotenziale in den drei Fallbereichen nutzbar gemacht werden können. Hierzu wurde in allen drei Fallbereichen auf Basis des im InGeMo-Projekt entwickelten SePIAR-Verfahrens (Gerlmaier 2020) bereichsspezifische Gestaltungsworkshops mit Führungskräften und ihren Teammitgliedern durchgeführt. 
Hierbei priorisierten die beteiligten Führungskräfte und Teammitglieder nach einer Bestandsaufnahme ihrer spezifischen digitalen Stressoren und möglichen Gestaltungspotenziale Handlungsschwerpunkte für ihren Arbeitsbereich (z.B. Reduzierung von Arbeitsbelastungen, Förderung des sozialen Miteinanders). Durch das Forschungsteam wurde den WorkshopTeilnehmenden im Rahmen entsprechender thematischer Workshop-Module arbeitswissenschaftlich fundiertes Gestaltungswissen für ihre gewählten Handlungsschwerpunkte vermittelt und mit ihnen konkrete Gestaltungsansätze für ihren Arbeitsbereich diskutiert und dokumentiert.

Im Anschluss daran erfolgte eine moderierte Zusammenführung der Gestaltungsvorschläge aus den Mitarbeiter*innen- und FührungskräfteWorkshops, in denen die Führungskräfte und Beschäftigten Umsetzungsmaßnahmen, Umsetzungsverantwortliche und Bearbeitungszeiten gemeinsam festlegten. In den Teams wurde im Anschluss daran vereinbart, den Stand der Bearbeitung in den regelmäßig stattfindenden Teamsitzungen zu besprechen. sechs Monate nach den kollektiven Festlegungen der Umsetzungsmaßnahmen wurde durch das Forschungsteam überprüft, welche der geplanten Gestaltungsmaßnahmen in den Teams umgesetzt werden konnten und ob sich die Gestaltungskompetenz und Gesundheitssituation der Teams verbessert hatte.

\section{Gestaltungserfolge im Umgang mit digitalen Stressoren}

Bei den umgesetzten Präventionsmaßnahmen in den Fallbereichen zeigte sich als auffälliger Befund, dass die Tätigkeitsbereiche mit überwiegender Facharbeit (Werkzeug) bzw. Wissensarbeit (Projektmanagement) mit elf bzw. zwölf umgesetzten Präventionsmaßnahmen deutlich besser abschnitten als im Fallbereich Adjustage mit überwiegend Anlerntätigkeiten (fünf Maßnahmen). Die Unterschiede im Umsetzungserfolg gingen einher mit erheblichen Differenzen bei den Aushandlungsprozessen zwischen Teammitgliedern und ihren Vorgesetzten in der Reflexionsphase:

Im Fallbereich Adjustage gelang es kaum, die unterschiedlichen Gestaltungsinteressen der Führungskräfte und Beschäftigten miteinander zu vereinbaren, wodurch nur Minimalkompromisse zustande kamen:

- Die Beschäftigten forderten zur Reduzierung der Arbeitsintensität einen zusätzlichen Kranführer, der Abteilungsleiter stellte im Folgenden jedoch nur ein Teammitglied für die Kranführung ab, stockte das Personal aber nicht auf.

- Die Beschäftigten hielten es für notwendig, zur besseren Beherrschung des Planungssystems umfangreiche Schulungen zu erhalten und das Planungstool ergonomisch zu überarbeiten. Der Abteilungsleiter wies aus Kostengründen jedoch nur die Schichtführer an, die Dateneingabe 
der Maschinenbediener vor Ort $\mathrm{zu}$ beobachten und gegebenenfalls nachträgliche individuelle Unterweisungen vorzunehmen.

- Die Beschäftigten wünschten sich zur Reduzierung ihrer Verspannungen und Rückenschmerzen ergonomische Verbesserungen wie vibrationsärmere Sitzgelegenheiten und eine bessere Beleuchtung. Der Abteilungsleiter wollte eine ergonomische Begutachtung im Rahmen einer 18 Monate später stattfindenden Sicherheitsbegehung abwarten und leitete stattdessen Rückenschulungstrainings durch eine Krankenkasse ein.

- Die Beschäftigten forderten zur Überwindung der technischen Ungewissheit mehr Transparenz über den Umgang mit den neu gewonnenen Betriebsdaten ein. Der Abteilungsleiter erklärte sich bereit, an den vierwöchentlich stattfindenden Schichtbesprechungen teilzunehmen und den aktuellen Stand der Revision zu berichten. In der Nachbesprechung wurde deutlich, dass die einzelnen Schichten das Angebot nicht wahrnahmen, weil sie sich von ihren Führungskräften nicht ernst genommen fühlten, wodurch sich das soziale Klima im Team weiter verschlechterte.

Im Gegensatz dazu gelang es im Fallbereich Werkzeug den Führungskräften und Beschäftigten nach einem Kompetenzaufbau im Rahmen der Gestaltungsworkshops deutlich besser, vorhandene Gestaltungskorridore zur Prävention im Arbeitsbereich zu nutzen:

- Zur Verminderung der Arbeitsintensität bzw. der hieraus resultierenden mentalen Überforderung bei den Programmierer*innen, in der Arbeitsvorbereitung und bei den Führungskräften wünschten sich die Mitarbeiter*innen die Möglichkeit, Blockzeiten für konzentrationsintensive Programmieraufgaben zu erhalten. Dies gewährte der Abteilungsleiter ebenso wie die Umfunktionierung eines Arbeitsraums in einen Ruhearbeitsraum.

- Zur Verminderung der Arbeitsüberlastung im Programmierteam und zur Verbesserung der Autonomie bei den Maschinenbedienern einigten sich die Abteilungsleitung und das Team auf eine fallweise Rückverlagerung der 2,5-D-Programmierung an die Maschinenoperateure.

- Zur Verbesserung der Transparenz von Prozessen in der Abteilung wurden regelmäßig stattfindende Teammeetings eingeführt, bei denen über neue Werkzeugprojekte informiert und über die Arbeitsauslastung der einzelnen Beschäftigten gesprochen werden konnte.

- Zur Reduzierung von Entgrenzungen zwischen Arbeiten und Freizeit führte die Abteilungsleitung auf Wunsch der Mitarbeitenden auch wie- 
der bedarfsweise bezahlte Schichtübergaben ein, um eine Erholung nach dem Arbeitsende zu gewährleisten.

- Um soziale Spannungen aufgrund technischer Veränderungen zukünftig besser bewältigen zu können, wurde mit den Führungskräften und Mitarbeitenden Strategien zum Deeskalationsmanagement erarbeitet und eingeübt.

- Die Abteilungsleitung gab den Mitarbeitenden zur Verbesserung der Erholungssituation eine explizite Erlaubnis, 5-minütige Kurzpausen nach etwa 90 Minuten Arbeitszeit durchzuführen. Auch wurde auf Wunsch der Mitarbeitenden für das gesamte Team ein Entspannungstraining durchgeführt und bestehende Räumlichkeiten als Pausenraum umfunktioniert.

Ähnlich wie im Bereich Werkzeug gelang es auch im Fallbereich Projektmanagement im Rahmen der Gestaltungsworkshops bestehende Gestaltungskorridore zur Stressverminderung zu identifizieren und Umsetzungsmaßnahmen kollektiv auszuhandeln:

- Zwar folgte der Abteilungsleiter den Teammitgliedern nicht in ihrem Wunsch, die Anzahl parallel zu bearbeitenden Projekte pro Person zu reduzieren. Er gewährte jedoch die Durchführung von sogenannten „stillen Stunden“ zu Förderung konzentrierten Arbeitens. Einige Subteams vereinbarten hierbei, sich morgens gegenseitig nicht bei der Arbeit zu unterbrechen und das Telefon lautlos zu stellen. Teammitglieder erhielten auch die Möglichkeit, sich für konzentrationsintensive Arbeiten an die Arbeitsplätze von auf Dienstreise befindlichen Vorgesetzten oder Kolleg*innen zu setzen.

- Zur Reduzierung von überlangen Arbeitszeiten und Zeitdruck setzte die Bereichsleitung darüber hinaus gegenüber einigen ihrer global agierenden Kunden den verstärkten Einsatz von Telefonkonferenzen durch, um häufige Dienstreisen zu vermeiden.

- Zur Erhöhung der persönlichen Zeitsouveränität und zur Ermöglichung von störungsfreiem Einarbeiten in neue Sachverhalte wurden umfangreiche Home-Office-Lösungen ermöglicht.

- Die Teamleiter unterstützten Mitarbeitende dabei, individuelle ergonomische Maßnahmen am Arbeitsplatz vorzunehmen, zum Beispiel das Aufstellen von Regalen zur Geräuschdämmung.

- Zur Verbesserung der Erholungssituation wurden die Teamleitungen durch den Abteilungsleiter angehalten, verstärkt auf die Einhaltung der Frühstücks- und Mittagspause sowie die Arbeitszeitlänge der Teammitglieder zu achten. 


\subsection{Gestaltungskompetenz und Ressourcenaufbau}

Im Rahmen der Evaluation ca. sechs bis acht Monate nach Abschluss der Workshops wurde auch untersucht, inwiefern in den drei Fallbereichen auf Basis der Gestaltungsworkshops ein Aufbau von kollektiver Gestaltungskompetenz stattgefunden hatte und ob sich hierdurch Verbesserungen bei Gesundheitsressourcen wie der erlebten Führung und sozialen Unterstützung oder einer Verminderung digitaler Stressoren ergeben hatte. Auswertungen der schriftlichen Vorher-Nachher-Befragungen von Führungskräften und Teammitgliedern ergaben, dass im Fall Werkzeug nach den Gestaltungsworkshops die Gestaltungskompetenz sowohl von Führungskräften als auch Beschäftigten deutlich gestiegen war. Im Bereich Projektmanagement fand sich ein sehr deutlicher Anstieg der Gestaltungskompetenz von Führungskräften bei einem stabil hohen Niveau der Gestaltungskompetenz ihrer Beschäftigten. Im Fallbereich Adjustage dagegen nahm im Zeitraum der Gestaltungsphase die Gestaltungskompetenz bei den Führungskräften ab, bei den Beschäftigten blieb sie auf niedrigem Niveau konstant (siehe Abbildung 1). 


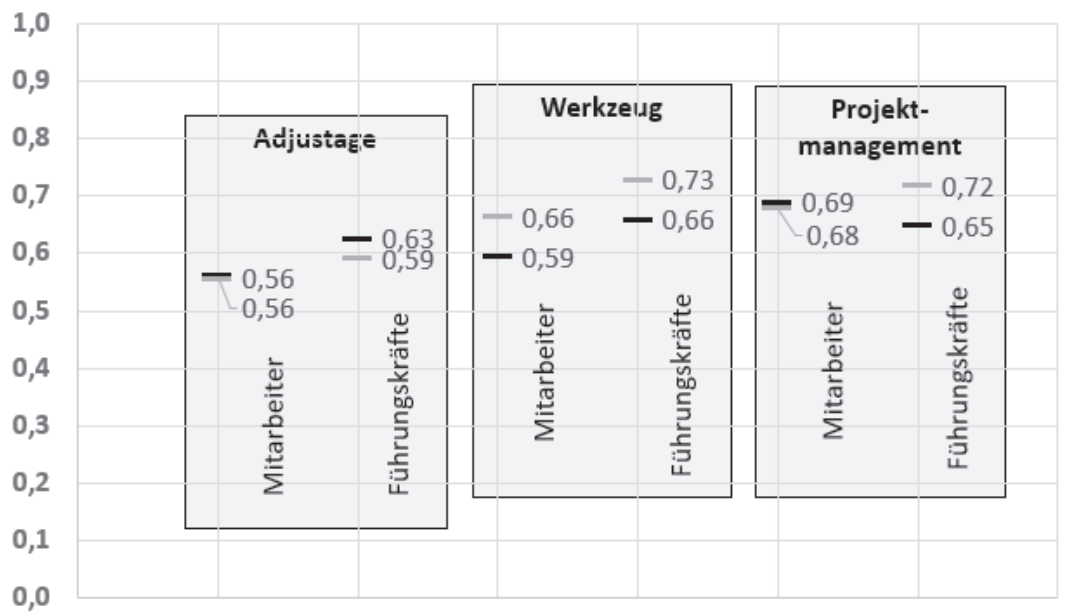

- Erstbefragung - Nachbefragung

Abbildung 1: Ausmaß der Gestaltungskompetenz im Vorher-Nachher-Vergleich für die drei Untersuchungsfälle getrennt nach Führungskräften und Beschäftigten. Wertebereich zwischen 0 (minimale Kompetenz) und 1 (maximale Kompetenz)

Neben den Veränderungen im Bereich der Gestaltungskompetenz erfolgte in den drei Fällen nach der Gestaltungsphase auch eine Betrachtung der Beanspruchungs- und Ressourcensituation. Hier zeigte sich, dass im Fall Projektmanagement und Werkzeug nach der Gestaltungsphase das Ausmaß der psychischen Beanspruchung gesunken war und sich die Ressourcensituation in den Untersuchungsfällen - insbesondere im Bereich der sozialen Unterstützung durch Vorgesetzte und der gesundheitsorientierten Führung - deutlich verbessert hatte. Dem entgegen ergab sich im Fallbereich Adjustage eine Verschlechterung der Beanspruchungswerte.

\section{Zusammenfassung und Diskussion}

Gegenstand des Beitrags war es zu hinterfragen, inwieweit die gegenwärtig in Industrieunternehmen stattfindenden Digitalisierungsprozesse Gestaltungsspielräume für eine menschengerechte Arbeitsgestaltung bieten und inwiefern diese derzeit von betrieblichen Gestaltungsakteuren genutzt werden. Bisher gibt es zur Frage gesundheitlicher Auswirkungen der Realisierung von Digitalisierungsprojekten in Produktionsunternehmen nur 
wenige empirische Feldstudien (u.a. Gerst 2011). Gegenstand der Diskurse stellen Bewertungen potenzieller Gesundheitsrisiken und -potenziale dar. Kontroversen bestehen aber auch darin, ob die digitale Produktion von morgen neue Gestaltungsrestriktionen oder Freiräume für präventive Arbeitsgestaltung (zum Beispiel durch taktungebundene Fließbänder, Assistenzsysteme) bewirkt (u.a. Kratzer 2018; Grote 2018). Im Beitrag wurde die These aufgestellt und anhand exemplarischer Fallstudien zu bekräftigen versucht, dass (erstens) neue Technologien nicht per se gesundheitsschädigend oder gesundheitsförderlich sind, sondern die Gestaltung der Arbeitssysteme hier eine bedeutende Rolle spielt. Digitalisierungsgewinne für Unternehmen und Beschäftigte lassen sich hierbei nur dann generieren, wenn Kriterien proaktiver und präventiver Arbeitsgestaltung von den betrieblichen Akteuren im Gestaltungsprozess frühzeitig berücksichtigt werden (Sträter und Bengler 2019; Anlauft et al. 2019). Entsprechend des Ansatzes der organisationalen Gestaltungskompetenz wurde darüber hinaus davon ausgegangen, dass (zweitens) der Erfolg des Gestaltungsprozesses wesentlich von der Gestaltungskompetenz der am Prozess beteiligten betrieblichen Akteure abhängig ist. Erfolgreiches betriebliches Präventionshandeln, so die dritte These, erfordert deshalb einen strategischen Aufbau von kollektiver Arbeitsgestaltungskompetenz und die Ermöglichung arbeitsimmanenter Lernprozesse im Rahmen des Digitalisierungsprozesses. Zur Prüfung dieser Thesen wurden exemplarisch drei von insgesamt acht im Forschungsprojekt durchgeführten Gestaltungsprojekte im Hinblick auf gesundheitliche Folgen nach Digitalisierungsvorhaben näher beleuchtet, wobei jeweils ein typischer Fall aus dem Bereich Anlerntätigkeiten, Facharbeit und produktionsnahe Wissensarbeit betrachtet wurde. Eine Verallgemeinerung der Befunde ist aufgrund der Fallzahl nicht geboten und nicht intendiert. Will man die Fälle im Hinblick auf proaktives Gestaltungshandeln bei der Technikeinführung in der Produktion deuten, so findet man in den unterschiedlichen Tätigkeitsfeldern vergleichbare Situationsschilderungen der Betroffenen im Hinblick auf das Beanspruchungserleben vor: die Beschäftigten aus unseren Beispielfällen brachten die Technologieeinführung in ihrem Arbeitsbereich mehrheitlich mit einer Zunahme von Stress in Verbindung, wenngleich die Belastungsmuster bei Anlerntätigkeiten, Facharbeit und produktionsnaher Wissensarbeit sich zum Teil deutlich voneinander unterschieden. Dies ging einher mit Selbsteinschätzungen der Führungskräfte, bei der Technikeinführung kaum arbeitswissenschaftliche Kriterien proaktiver Arbeitsgestaltung berücksichtigt zu haben. Ähnliche Ergebnisse berichten Georg et al. (2017). Ansätze proaktiven Gestaltungshandelns fanden sich in unseren drei Fallbereichen beim Informationsmanagement und zum Teil im Bereich der Qualifizierung zur För- 
derung der technischen Beherrschbarkeit bei den Beschäftigten. Vorausschauende Aspekte des Arbeits- und Gesundheitsschutzes oder persönlicher Schutzrechte bei der Sammlung von Betriebsdaten spielten in allen drei Fallbereichen bei der Technikeinführung jedoch eine untergeordnete Rolle.

Gegenstand des Beitrags war es auch zu untersuchen, welche Gestaltungschancen für gesunde Arbeit sich im realen betrieblichen Produktionskontexten bei Anlerntätigkeiten, Facharbeit und produktionsnaher Wissensarbeit bei der Einführung von Digitaltechnik ergeben und wie diese Potenziale erschlossen werden können. Untersucht wurde dabei speziell, welchen Beitrag ein Aufbau lokaler und kollektiver Gestaltungskompetenz dazu leisten kann. Hier zeigte sich, dass in den drei Untersuchungsbereichen Arbeitssystem bedingt unterschiedlich große und in der Qualität variierende Gestaltungskorridore vorlagen (z.B. Home-Office im Bereich Projektmanagement oder Ruhearbeitsplätze im Bereich Werkzeug). Die Nutzung der Gestaltungskorridore gelang aber insbesondere in Fallbereichen, bei denen die Führungskräfte und Beschäftigten sich im Laufe der Gestaltungsworkshops erweiterte Arbeitsgestaltungskompetenzen aneigneten und motiviert waren, erlerntes Gestaltungswissen in ihrer konkreten Arbeitssituation anzuwenden.

Diese Beobachtungen aus den Gestaltungsworkshops spiegeln sich auch in den Ergebnissen aus der Evaluationsphase wider: Im Bereich Werkzeug und Projektmanagement ging die große Anzahl erfolgreich umgesetzter Präventionsmaßnahmen einher mit einer deutlichen Steigerung der Arbeitsgestaltungskompetenz bei den operativen Führungskräften und Beschäftigten (gemessen sechs Monate nach Abschluss der Maßnahmen). Im Fallbereich Adjustage erwies sich insbesondere die geringe Gestaltungsmotivation der Bereichsleitung als Hemmfaktor für die Umsetzung von nachhaltigen Präventionsmaßnahmen. Letzteres verdeutlicht einmal mehr, dass Gestaltungchancen nicht nur durch technische Voraussetzungen, sondern auch von Kompetenzen und Verwertungsstrategien verhandlungsmächtiger Führungskräfte abhängig sind. Auf Befähigung und Beteiligung setzende Vorgehensmodelle, wie sie im Forschungsprojekt angewendet wurden, sind in diesen Fällen begrenzt wirksam und zeigen an dieser Stelle die Notwendigkeit von Regulierung für einen Interessenausgleich auf. Hieraus folgt, dass es zur Umsetzung proaktiver Gestaltungstrategien im Betrieb mehrerer aufeinander aufbauender Gelingensfaktoren bedarf. Dazu zählt zum einen die Befähigung und direkte Beteiligung der verschiedenen Akteursgruppen vor Ort. Ebenso wie eine betriebliche Präventionsstrategie mit verbindlichen Arbeitsschutzstrukturen und Verfahrensregelungen zum Interessenausgleich. Konkret haben sich zur Ausbildung einer proak- 
tiven Gestaltungstrategie bei Transformationsprozessen folgende Erfolgsfaktoren herauskristallisiert:

Erstens: proaktives Gestaltungshandeln erfordert die Ausbildung eines kollektiven Problembewusstseins über Gesundheitsrisiken und Gestaltungspotenziale bei Digitalisierungsprozessen. Eine kontinuierliche Sensibilisierung aller Akteursgruppen im Betrieb - etwa durch regelmäßig stattfindende Unterweisungen (digital oder face to face) oder andere Formen des Informationsmanagements - ist hilfreich, um psycho-soziales Gefahren-bzw. Gestaltungswissen aufzubauen und zu etablieren. Uninformiertheit einzelner Akteursgruppen hingegen führt oft $\mathrm{zu}$ unproduktiven Handlungsblockaden.

Zweitens: digitale Transformationsprojekte verlaufen umso erfolgreicher, wenn davon lokal betroffene operative Führungskräfte und Beschäftigte frühzeitig in den Planungs- bzw. Gestaltungsprozessen aktiv beteiligt werden. Der Aufbau und konsequente Einsatz partizipativer und kompetenzstärkender Vorgehensmodelle bei betrieblichen Transformationsprozessen ist hilfreich, um sowohl das Prozesswissen vor Ort als auch spezifische Bedürfnisse und Interessenlagen von Führungskräften und Beschäftigten auszutarieren.

Drittens: hoch vernetzte und fragile Produktionssysteme erfordern einen systematischen Aufbau von Reflexionsräumen zum Dialog. Partizipativ angelegte regelmäßige Gefährdungsbeurteilungen wie auch kurzzyklische Teammeetings, bei denen Arbeitsbelastungen einen regelmäßig wiederkehrenden Tagesordnungspunkt darstellen, erwiesen sich als sehr effizient, um Belastungspotenziale zeitig zu erkennen und unmittelbar anzugehen.

Viertens: eine funktionsfähige Arbeitsschutzstruktur mit kompetenten Arbeitsschutz-Professionals und Interessenvertretungen stellen gute Voraussetzung dar, damit Digitalisierungsprojekte frühzeitig im Hinblick auf Kriterien menschengerechter Arbeit geplant und umgesetzt werden.

Grundsätzlich deuten die im Rahmen der Gestaltungsprojekte erzielten Präventionserfolge darauf hin, dass Digitalisierungsvorhaben in der Produktion und den produktionsnahen Servicebereichen durchaus Chancen für eine präventive und proaktive Arbeitsgestaltung in sich bergen. Die Nutzung von Gestaltungchancen wird jedoch nur dann erfolgen, wenn die verschiedenen betrieblichen Akteursgruppen über ArbeitsgestaltungsKnow-how verfügen und dieses frühzeitig in den Planungs- und Gestaltungsprozess einbringen können. Schwierigkeiten dieses Befähigungsansatzes existieren offenbar vermehrt bei unqualifizierten Beschäftigtengruppen. Die regelmäßige Durchführung vom Gesetzgeber eingeforderter Gefährdungsbeurteilungen kann hier hilfreich sein, eine weitere drohende 
Kumulation von Fehlbelastungen zu vermeiden. Verstärkte Beratungsund Überwachungsaktivitäten der (aber personell derzeit unterbesetzten) staatlichen Arbeitsschutzinstitutionen könnten dazu beitragen, die derzeitig mit $30 \%$ geringe Umsetzungsquote in den Betrieben deutlich zu erhöhen (Urban 2019).

\section{Literatur}

Ahlers, E. (2018). Die Digitalisierung der Arbeit. Verbreitung und Einschätzung der Betriebsräte. WSI-Report Nr. 40. Düsseldorf: Hans-Böckler-Stiftung.

Anlauft W., Habenicht T., \& Klippert J. (2019). Arbeit 4.0 - Proaktive Arbeitsgestaltung als ein zentrales Handlungsfeld für die betriebliche Interessenvertretung. In A. Gerlmaier \& E. Latniak (Hrsg.) (2019), Handbuch psycho-soziale Gestaltung digitaler Produktionsarbeit. Wiesbaden: Springer Gabler.

Backhaus, N. (2018). Review zur Wirkung elektronischer Überwachung am Arbeitsplatz und Gestaltung kontextsensitiver Assistenzsysteme. Dortmund, Berlin, Dresden: Bundesanstalt für Arbeitsschutz und Arbeitsmedizin.

Bakker, A. B., \& Demerouti, E. (2007). The Job Demands-Resources model: state of the art. Journal of Managerial Psychology, 22(3), 309-328.

Bamberg E., \& Busch C. (2006). Stressbezogene Interventionen in der Arbeitswelt. Zeitschrift für Arbeits- und Organisationspsychologie, 50(4), 215-226.

BAuA (2020) Volkswirtschaftliche Kosten durch Arbeitsunfähigkeit 2018. Verfügbar unter: https://www.baua.de/DE/Themen/Arbeitswelt-und-Arbeitsschutz-imWandel/Arbeitsweltberichterstattung/Kosten-der-AU/pdf/Kosten-2018.pdf?_blo $\mathrm{b}=$ publicationFile\&v=3 [23.09.2020].

BDA (2015). Chancen der Digitalisierung nutzen. Positionspapier der BDA zur Digitalisierung von Wirtschaft und Arbeitswelt. Verfügbar unter: https://arbeitgeb er.de/www/arbeitgeber.nsf/res/6308287022D75F36C1257FA2005707DD/\$file/B DA_Chancen_Digitalisierung.pdf [23.09.2020].

Böhm, S. A., Bourovoi, K., Brzykcy, A. Z., Kreissner, L. M., \& Breier, C. (2016). Auswirkungen der Digitalisierung auf die Gesundheit von Berufstätigen: Eine bevölkerungsrepräsentative Studie in der Bundesrepublik Deutschland. St. Gallen: Universität St. Gallen.

Derks, D., ten Brummelhuis, L. L., Zecic, D., \& Bakker, A. B. (2012). Switching on and off ...: Does smartphone use obstruct the possibility to engage in recovery activities? European Journal of Work and Organizational Psychology, 23(1), 1-11.

Dettmers, J., Vahle-Hinz, T., Friedrich, N., Keller, M., Schulz, A., \& Bamberg, E. (2012). Entgrenzung der täglichen Arbeitszeit - Beeinträchtigungen durch ständige Erreichbarkeit bei Rufbereitschaft. In B. Badura, A. Ducki, H. Schröder, J. Klose \& M. Meyer (Hrsg.), Feblzeiten-Report 2012 (S. 53-60). Berlin, Heidelberg: Springer. 
DGB (Institut DGB-Index Gute Arbeit) (2017). Verbreitung, Folgen und Gestaltungsaspekte der Digitalisierung in der Arbeitswelt - Auswertungsbericht auf Basis des DGB-Index Gute Arbeit 2016. Berlin: DGB.

DGUV (2017). Industrie 4.0: Herausforderungen für die Prävention - Positionspapier der gesetzlichen Unfallversicherung. Verfügbar unter: https://www.dguv.de /medien/inhalt/praevention/arbeitenvierpunktnull/pospap-2-2017.pdf [23.09.2020].

Dickens, L., \& Watkins, K. (1999). Action research: Rethinking Lewin. Management Learning, 30(2), 127-140.

Ducki, A., \& Nguyen, H. T. (2016). Psychische Gesundheit in der Arbeitswelt-Mobilität. Dortmund: Bundesanstalt für Arbeitsschutz und Arbeitsmedizin.

Erpenbeck, J., \& von Rosenstiel, L. (2007). Handbuch Kompetenzmessung: erkennen, verstehen und bewerten von Kompetenzen in der betrieblichen, pädagogischen und psychologischen Praxis (2. erw. Auflage). Stuttgart: Schäffer-Poeschel.

Faber, U., \& Satzer, R. (2016). Arbeitsschutz und Mitbestimmung - Handlungshilfe für Betriebsräte. Reihe: IG BCE Stichworte 16. Frankfurt: Bund-Verlag.

Georg, A., Katenkamp, O., \& Guhlemann, K. (2017). Digitalisierungsprozesse und das Handeln von Betriebsräten: Strategien und Handlungsoptionen von Betriebsräten in der Arbeitswelt 4.0. Arbeit, 26(2), 251-274.

Gerlmaier, A. (2020). Mit kollektiver Gestaltungskompetenz Stress im Team begegnen: das SePIAR-Qualifizierungskonzept. Betriebliche Prävention, 132(1), 10-15.

Gerlmaier, A. (2018a). Organisationale Gestaltungskompetenz im Betrieb - ein (unterschätzter) Mediator des Zusammenhangs von psychischer Belastung und Beanspruchung. Zeitschrift für Arbeitswissenschaft, 72(2), 130-136.

Gerlmaier, A. (2018b). Gesund arbeiten im Zeitalter der Digitalisierung: Wo liegen die Arbeitsgestaltungspotenziale aus Sicht von Beschäftigten und Unternehmensvertretern? IAQ-Report Nr. 2018-04. Duisburg: Institut Arbeit und Qualifikation.

Gerlmaier, A., \& Latniak, E. (2013). Psychische Belastungen in der IT-Projektarbeit - betriebliche Ansatzpunkte der Gestaltung und ihre Grenzen. In G. Junghanns \& M. Morschhäuser (Hrsg.), Immer schneller, immer mehr: psychische Belastung bei Wissens- und Dienstleistungsarbeit. Bundesanstalt für Arbeitsschutz und Arbeitsmedizin (BAuA) (S. 165-193). Wiesbaden: Springer VS.

Gerst D. (2011). Sind ganzheitliche Produktionssysteme und Gute Arbeit vereinbar? Arbeit, 20(3), 246-250.

Gimpel, H., Lanzl, J., Manner-Romberg, T., \& Nüske, N. (2018). Digitaler Stress in Deutschland. Eine Befragung von Erwerbstätigen zu Belastung und Beanspruchung durch Arbeit mit digitalen Technologien. Forschungsförderung Working Paper, Nr. 101. Düsseldorf: Hans-Böckler-Stiftung.

Grote, G. (2018). Gestaltungsansätze für das komplementäre Zusammenwirken von Mensch und Technik in Industrie 4.0. In H. Hirsch-Kreinsen, P. Ittermann \& J. Niehaus (Hrsg.), Digitalisierung industrieller Arbeit. Die Vision Industrie 4.0 und ibre sozialen Herausforderungen (S. 215-232). Baden-Baden: Nomos.

Haipeter, T. (2019). Interessenvertretung in der Industrie 4.0. Das gewerkschaftliche Projekt Arbeit 2020. Baden-Baden: Nomos. 
Hammer, S., Deitermann, M., Bradaran, G., Siedler, S., Bugge, K., \& Haas, C. T. (2019). Gesundheit und Gesundheitsförderung am Arbeitsplatz aus Mitarbeiterperspektive. Zeitschrift für Arbeitswissenschaft, 73, 295-311.

Hirsch-Kreinsen, H. (2018). Die Pfadabhängigkeit digitalisierter Industriearbeit. Arbeit, 27(3), 239-259.

Hirsch-Kreinsen, H. (2017). Digitalisierung industrieller Einfacharbeit. Arbeit, 26(1), 7-32.

Heidenreich, M., Kirch, B., \& Mattes, J. (2008). Die organisatorische Einbettung von Informationstechnologien in einem globalen Entwicklungsprojekt. In C. Funken \& I. Schulz-Schaeffer (Hrsg), Digitalisierung der Arbeitswelt: Zur Neuordnung formaler und informeller Prozesse in Unternebmen (S. 193-220). Wiesbaden: Springer VS.

Höge, T., \& Büssing, A. (2004). The Impact of Sense of Coherence and Negative Affectivity on the Work Stressor-Strain Relationship. Journal of Occupational Health Psychology, 9(3), 195-205.

Huchler, N. (2019). Assimilierende versus komplementäre Adaptivität. In H. Hirsch-Kreinsen \& A. Karacic (Hrsg.), Autonome Systeme und Arbeit. Perspektiven, Herausforderungen und Grenzen der Künstlichen Intelligenz in der Arbeitswelt (S. 139-180). Bielefeld: transcript.

Janneck, M., \& Hoppe, A. (Hrsg.) (2018). Gestaltungskompetenzen für gesundes Arbeiten - Arbeitsgestaltung im Zeitalter der Digitalisierung. Berlin: Springer.

Junghanns, G., \& Kersten, N. (2019). Informationsflut durch digitale Medien am Arbeitsplatz. Bericht zum 65. Arbeitswissenschaftlichen Kongress vom 27. Februar-1. März 2019 in Dresden. Zentralblatt für Arbeitsmedizin, Arbeitsschutz und Ergonomie, 69(3), 119-132.

Kratzer N. (Hrsg.) (2018). Arbeit-der-Zukunft. Digital, multilokal, dynamisch - Thesen und Gestaltungsansätze für den Arbeitsplatz der Zukunft. Broschüre der Fokusgruppe „Gesunde Arbeit durch innovative Arbeitsformen und Assistenzsysteme. Siegen.

Lechleiter, P., Purbs, A., \& Sonntag, K. (2019). HR-und Gesundheitsmanagement in der Arbeit 4.0 - Bedarfe in deutschen und internationalen Unternebmen (Kurzfassung). Heidelberg: Uni Heidelberg.

Lenhardt, U. (2017). Psychische Belastung in der betrieblichen Praxis - Erfahrungen und Sichtweisen präventionsfachlicher Berater. Zeitschrift für Arbeitswissenschaft, 71, 6-13.

Lück, M., Hünefeld, L., Brenscheidt, S., Bödefeld, A., \& Hünefeld, A. (2019). Grundauswertung der BIBB/BAuA-Erwerbstätigenbefragung 2018 - Vergleich zur Grundauswertung 2006 und 2012. Forschung Projekt F 2417M. Dortmund: Bundesanstalt für Arbeitsschutz und Arbeitsmedizin.

Maier, C., Laumer, S., \& Weinert, C. (2015). Enterprise Resource Planning Systems Induced Stress: a Comparative Empirical Analysis with Young and Elderly SAP Users. Wirtschaftsinformatik Proceedings, Paper 93. 
Richenhagen, G., \& Dick, M. (2019). Aktions- und Handlungsforschung in den Arbeitswissenschaften. In GfA (Hrsg.), Arbeit interdisziplinär analysieren - bewerten - gestalten. Dokumentation des 65. Arbeitswissenschaftlichen Kongresses an der Technischen Universität Dresden vom 27.02.-01.03.2019. Beitrag C.8.7. Dortmund: GfA-Press.

Robelski, S. (2016). Psychische Gesundheit in der Arbeitswelt - Mensch-Maschine-Interaktion. Forschung F2353. Berlin: Bundesanstalt für Arbeitsschutz und Arbeitsmedizin (BAuA).

Rothe, I., Adolph, L., Beermann, B., Schütte, M., Windel, A., Grewer, A., Lenhardt, U., Michel, J., Thomson, B., \& Formazin, M. (2017). Psychische Gesundheit in der Arbeitswelt - Wissenschaftliche Standortbestimmung. Dortmund: Bundesanstalt für Arbeitsschutz und Arbeitsmedizin.

Schempp, N., \& Strippel, H. (2017). Präventionsbericht 2017. Berlin, GKV Spitzenverband. Verfügbar unter: https://www.gkv-spitzenverband.de/media/dokument e/krankenversicherung_1/praevention_selbsthilfe__beratung/praevention/prae ventionsbericht/2017_GKV_MDS_Praeventionsbericht.pdf [23.09.2020].

Schmidt, F. A. (2017). Digital Labour Markets in the Platform Economy. Mapping the Political Challenges of Crowd Work and Gig Work. Bonn: Friedrich-Ebert-Stiftung.

Sträter, O., \& Bengler, K. (2019). Positionspapier Digitalisierung der Arbeitswelt. Zeitschrift für Arbeitswissenschaft, 73, 243-245.

Trägner, U. (2006). Arbeitszeitschutzrechtliche Bewertung der Intensität von Arbeitsleistungen unter besonderer Berücksichtigung der Rechtsprechung des Europäischen Gerichtshofes zum Bereitschaftsdienst. Konstanz: Hartung-Gorre.

Urban, H.J. (2016). Arbeiten in der Wirtschaft 4.0 - Über kapitalistische Rationalisierung und digitale Humanisierung. In L. Schröder \& H.J. Urban (Hrsg.), DGB Jabrbuch Gute Arbeit 2016 - Digitale Arbeitswelt (S. 21-45). Frankfurt am Main: DGB.

Urban, H.-J. (2019). Aufsicht am Limit. Gute Arbeit kompakt, Ausgabe Nr. 11 /2019.

Weiß, C. (2012). Subjektivierte Selbstsorge als Bewältigungsstrategie. In B. Badura, A. Ducki, H. Schröder, J. Klose \& M. Meyer (Hrsg.), Fehlzeiten-Report 2012: Gesundheit in der flexiblen Arbeitswelt: Chancen nutzen - Risiken minimieren (S. 203209). Heidelberg: Springer.

Zimmermann K. (2017). Digitalisierung der Produktion durch Industrie 4.0 und ihr Einfluss auf das Arbeiten von morgen. In B. Spieß \& N. Fabisch (Hrsg.), CSR und neue Arbeitswelten: Perspektivwechsel in Zeiten von Nachhaltigkeit, Digitalisierung und Industrie 4.0 (S. 53-72). Heidelberg: Gabler. 
